\title{
Correlation characteristics assessment of construction products reliability parameters
}

\author{
Nadezhda Chiganova* \\ Moscow State University of Civil Engineering, Yaroslavskoe shosse, 26, Moscow, 129337, Russia
}

\begin{abstract}
Since the reliability of construction products, by definition, is a probability of failure-free operation, then to assess the correlation characteristics between the parameters of the reliability of construction products that are not observed at the same time, we will use the designations and terms used in the theory of probability and mathematical statistics. Existing statistical methods for assessing the conditional mathematical expectation applied to the construction product reliability are based on the possibility of simultaneous measurement of the value of random variables. Because of this, they get a set of extreme points in the plane of those variables, and then build a regression line estimates based on those parameters. Such methods are obviously unacceptable for the case when the quantities the mentioned random variables are cumulatively unobserved. The solution to this problem will allow us to find estimates (not statistical) of the regression line and a number of other characteristics associated with it for simultaneously unobservable random variables. The algorithm described in the current work illustrates an example of interest in the theory of reliability for finding the extrema of the averaged function over the given initial parameters of the construction product.
\end{abstract}

\section{Introduction}

Since the reliability of construction products, by definition, is a probability of failure-free operation, then to assess the correlation characteristics between the parameters of the reliability of construction products that are not observed at the same time, we will use the designations and terms used in the theory of probability and mathematical statistics. By a 'construction product' in the current work we will assume any given material, product or construction with a set of known parameters, obtained during the initial testing, that can be described using mathematical functions [1-5], applicable in reliability theory.

Let us assume $\xi$ and $\eta$ be two dependent random variables with joint distribution set as $K(x, y)=P(\xi<x, \eta<y)[4,6]$

It is assumed that the function $\mathrm{K}$ is unknown, and only the distributions $F(x)=P(\xi<$ $x)$ и $G(y)=P(\eta<y)$ are given. Since it is impossible to recover the function $\mathrm{K}$ from the one-dimensional distributions $\mathrm{F}$ and $\mathrm{G}$ [5], the conditional mathematical expectation

*Corresponding author: chiganovanm.mgsu@gmail.com 
$I_{Q}(x)=M_{x} \varphi(y)=\int \varphi(y) d Q(y / x)$ of the known function $\varphi$ (y) is determined ambiguously by specifying $\mathrm{F}$ and $\mathrm{G}$ [5].

Here $Q(y / x)=P(\eta<y / \xi<x)$.

In [5], lower and upper bounds for $I_{Q}(x)$ are found under the assumption that $I_{Q}(x)$ is a non-decreasing function in $\mathrm{x}$ [6-8]. In this work, the extremum of another function is determined $R_{Q}(\Pi, F, G)=\int I_{Q}(x) d \Pi(x)$, which is obtained by averaging $I_{Q}(x)$ over the given distribution $\Pi(x)$. We denote $\mathrm{L}(\mathrm{F}, \mathrm{G})$ as the set of all conditional distributions $\mathrm{Q}$ for which $I_{Q}(x)$ is a non-decreasing function in $\mathrm{x}$, and $\bar{m}(F, G)=\left\{Q: \int Q(y / x) d F(x)=\right.$ $G(y)\}$

Assuming all stated above, the problem under consideration can be formulated as follows. It is required to find the absolute minimum $m(\Pi, F, G)$ and absolute maximum $M(\Pi, F, G)$ of the function $R_{Q}(\Pi, F, G)$ on the set $S(F, G) \in L(F, G) \cap \bar{m}(F, G)[3,5-8]$

The solution to this problem is of interest in the theory of reliability [5,7], since it can be used to estimate the regression line between simultaneously unobservable random variables. Indeed, for $\varphi(y)=y$, the characteristic $I_{Q}(x)$ is a regression line between $\xi$ and $\eta$. Existing statistical methods for assessing $I_{Q}$ are based on the possibility of simultaneous measurement of the value of random variables $\xi$ and $\eta[1,3,6-13]$. Because of this, they get a set of extreme points $\left(\xi_{1}, \eta_{1}\right)\left(\xi_{2}, \eta_{2}\right) \ldots\left(\xi_{\mathrm{n}}, \eta_{\mathrm{n}}\right)$ in the plane $(\xi, \eta)$, and then build a regression line estimates based on those parameters. Such methods are obviously unacceptable for the case when the quantities $\xi$ and $\eta$ are cumulatively unobserved. An example of such quantities are the moments of failure of a construction product in various modes [6-13].

The solution to this problem will allow us to find estimates (not statistical) of the regression line and a number of other characteristics associated with it for simultaneously unobservable random variables. The initial data for these estimates are the distributions $\mathrm{F}$ and $\mathrm{G}$, and the monotonicity of the function $I_{Q}(x)$ (regression lines). The distributions of $\mathrm{F}$ and $\mathrm{G}$ can be determined from experimental data, and the monotonicity of $I_{Q}(x)$ often follows from physical considerations.

\section{Methods}

In what follows, we restrict ourselves to considering the case when $\xi$ takes on a finite number of values $\mathrm{X}=\left(x_{1}, x_{2}, \ldots, x_{n}\right)$, and distribution $\Pi(x)$ is focused on multiple $\mathrm{X}[5,6-$ $8,10,11]$. In the discrete case, it is convenient to write down the distribution $\mathrm{Q}(\mathrm{y} / \mathrm{x})$ in the form of a column matrix $Q=\left(Q_{1}, Q_{2}, \ldots, Q_{n}\right)^{T}, Q_{i}=Q\left(y / x_{i}\right)$, and instead of the distributions $F$ and $G$, introduce, respectively, the sets $\left(\alpha_{1}, \alpha_{2}, \ldots, \alpha_{n}\right)$ и $\left(\beta_{1}, \beta_{2}, \ldots, \beta_{n}\right)$, where $\alpha_{i}$ and $\beta_{i}$ are the magnitude of the jump at the point $\mathrm{x}$, respectively, of the functions $\mathrm{F}$ and $\Pi$. It is obvious, that $\alpha_{i}>0, \beta_{i} \geq 0, \alpha_{1}+\alpha_{2}+\ldots+\alpha_{n}=1, \beta_{1}+\beta_{2}+\ldots+\beta_{n}=1$.

By using the following statements as a theoretical foundation we can further analyze the involvement of certain parameters in the reliability assessment process of the construction products.

\section{Results}

The main result of the current work is contained in the following theorem.

We introduce the concept of limit groups and limit distributions $\Pi^{*}$ and $F^{*}$ accordingly. Let us assume $\gamma_{i}=\beta_{i} / \alpha_{i}$. Using $\gamma_{i}$ we can distinguish all groups. Let us agree to denote them as $X_{i}^{(1)}, i=1,2, \ldots, k_{1}, k_{1} \leq n$, and $\alpha_{i}^{(1)}=\sum_{x_{j} \in X_{i}^{(1)}}^{i} \alpha_{j}\left(x_{j}\right), \beta_{i}^{(1)}=\sum_{x_{j} \in X_{i}^{(1)}}^{i} \beta_{j}\left(x_{j}\right), j=$ 
$1,2, \ldots, n$, corresponding with samples $\left\{\alpha_{i}^{(1)}\right\}$ and $\left\{\beta_{i}^{(1)}\right\}$. Distribution functions will be denoted as $\Pi^{(1)}$ and $F^{(1)}$ accordingly.

Using $\gamma_{i}^{(1)}=\beta_{i}^{(1)} / \alpha_{i}^{(1)}$ let us distinguish all the groups $X_{i}^{(2)}, i=1,2, \ldots, k_{2}, k_{2} \leq k_{1}$.

Applying this procedure several times, we obtain the distribution functions for $N \leq n$ $\gamma_{1}^{(N)} \geq \gamma_{2}^{(N)} \geq \ldots \geq \gamma_{k}^{(N)}$.

In subsequent steps $X_{i}^{(N)}$ will not change. We will name $X_{i}^{(N)}$ the limit groups, and $\gamma_{i}^{(N)}$ the limit probability ratio and denote them as $X_{i}^{*}$ and $\gamma_{i}^{*}$ accordingly. Also, we name $\Pi_{(x)}^{*}$ and $F_{(x)}^{*}$ as limit distribution functions, corresponding with probabilities $\alpha_{i}^{*}=\sum_{x_{j} \in X_{i}^{*}} \alpha_{j}\left(x_{j}\right)$ $\beta_{i}^{*}=\sum_{x_{j} \in X_{i}^{*}} \beta_{j}\left(x_{j}\right)$.

Let us analyze the following theorem.

The absolute minimum of the $R_{Q}$ function is reached on the distribution

$$
Q^{*}(y / x)=G(y) \mid \begin{aligned}
& F^{*}(x+0) \\
& F^{*}(x-0)
\end{aligned}
$$

The proof follows from the statement that $\inf _{Q \in S\left(\Pi^{*}, G\right)} R_{Q}\left(\Pi^{*}, F^{*}, G\right)=\inf _{Q \in S(F, G)} R_{Q}(\Pi, F, G)$.

Absolute maximum of the $R_{Q}(\Pi, F, G)$ function is found according to the same algorithm, assuming the selection of groups should be made according to the criterion $\gamma_{1} \leq \gamma_{2}$.

Let us briefly present an algorithm for finding the extrema of the function $R_{Q}$, which is as follows. The ratio of probabilities $\gamma(0)=\infty, \mathrm{i}=1,2, \ldots, \mathrm{n}, \gamma_{n+1}=0$ is compiled.

If for some numbers $\gamma_{e-1}>\gamma_{e} \leq \gamma_{e+1} \leq \ldots \leq \gamma_{e+k}>\gamma_{e+k+1}$, then instead of using $\gamma(x)$ at the first step, a new probability ratio is introduced:

$$
\gamma^{(1)}(x)=\left\{\begin{array}{c}
\gamma(x), x \bar{\in}\left(x_{e}, x_{e+1}, \ldots, x_{e+k}\right) \\
\frac{\beta_{e}+\ldots+\beta_{e+k}}{\alpha_{e}+\ldots+\alpha_{e+k}}, x \in\left(x_{e}, x_{e+1}, \ldots, x_{e+k)}\right.
\end{array}\right.
$$

If it turns out again that for some numbers $\gamma_{\delta-1}^{(1)}>\gamma_{\delta}^{(1)} \leq \gamma_{\delta+1}^{(1)} \leq \ldots \leq \gamma_{\delta+m}^{(1)}>\gamma_{\delta+m+1}^{(1)}$, then instead of using $\gamma^{(1)}(x)$ a new probability ratio is introduced:

$$
\gamma^{(2)}(x)=\left\{\begin{array}{c}
\gamma^{(1)}(x), x \bar{\epsilon}\left(x_{\delta}, x_{\delta+1}, \ldots, x_{\delta+m}\right) \\
\frac{\beta_{\delta}+\ldots+\beta_{\delta+m}}{\alpha_{\delta}+\ldots+\alpha_{\delta+m}}, x \in\left(x_{\delta}, x_{\delta+1}, \ldots, x_{\delta+m}\right)
\end{array}\right.
$$

and so on. This process continues until a non-returning function is obtained at some step $\gamma(x)$.

It was proved [14] that thus $\gamma(\mathrm{x})$ is uniquely determined.

Using $\gamma(\mathrm{x})$, the extremal distribution $Q^{*}(y / x)$, at which the absolute minimum of the function $R_{Q}$ is attained, can be written in the form:

The following notations are used here:

$$
Q^{*}(y / x)=\omega(y) \mid \begin{aligned}
& F(x+0) \\
& F(x-0)
\end{aligned}
$$

$$
\begin{gathered}
F(x)=\int_{\gamma(\xi)>\gamma(x)} d F(\xi) \\
\omega(y)=\int_{I(\eta)<y} d G(\eta)
\end{gathered}
$$

$\left.\omega\right|_{\alpha} ^{\beta}=(\beta-\alpha)^{-1} \omega(y-\alpha)$ is the interval-centered truncated distribution $\left[y_{1}, y_{2}\right]$, where $\mathrm{y}_{1}$ and $\mathrm{y}_{2}$ are the solution of the system of inequalities:

$$
\omega\left(y_{1}+0\right) \geq \alpha
$$




$$
\begin{aligned}
& \omega\left(y_{1}-0\right) \leq \alpha \\
& \omega\left(y_{2}+0\right) \leq \beta \\
& \omega\left(y_{2}-0\right) \geq \beta
\end{aligned}
$$
$\mathrm{y}_{2}$.

Function $\left.G\right|_{\alpha} ^{\beta}$ is uniquely determined regardless of the chosen solution of the system $\mathrm{y}_{1}$,

The extremal distribution $\mathrm{Q}$, at which the absolute maximum of the function $\mathrm{R}$ is achieved, is found in a similar way [14-16]. The only difference is that when constructing a sequence of functions $\gamma^{(1)}, \ldots, \gamma^{(n)}$, it is necessary to change the signs of the inequalities to the opposite.

The described algorithm for finding the extrema of the $R_{Q}$ function illustrates an example of interest in the theory of reliability [15-18].

\section{Discussion}

As an example of the algorithm application let us consider the following problem.

Let construction products with a certain probability be tested in one of ten modes described by the parameter $\mathrm{x}_{\mathrm{i}}, i=1,2, \ldots, 10, x_{i} \leq x_{i+1}$

Let us suppose, that it is known that when the choice of the mode $x_{i}$ is carried out with the probability $\alpha_{\mathrm{i}}=0,1 ; i=1,2, \ldots, 10$, the failure rate $\lambda$ of construction products is constant in time and equal to $\frac{1}{\mathrm{~T}}$, where $\mathrm{T}=1000$ hours. The objective is to estimate the mean time $\mathrm{R}$ of no-failure operation of construction products, if they are tested in mode $\mathrm{x}_{\mathrm{i}}$ with probability $\beta_{i}$, moreover, in our case $\beta_{1}=\beta_{2}=\beta_{3}=\beta_{4}=\beta_{6}=\beta_{8}=0,1 ; \beta_{5}=\beta_{10}=$ 0,$05 ; \beta_{7}=\beta_{9}=0,15[17,19]$.

Note that the function $Q\left(y / x_{i}\right)$, which is the probability of failure of a construction product in time $\mathrm{y}$ in the mode $\mathrm{x}_{\mathrm{i}}$, is assumed to be unknown, but from physical considerations the following inequality is considered to be satisfied: $I_{Q}\left(x_{i}\right) \leq I_{Q}\left(x_{i+1}\right), \mathrm{i}=1$, $2, \ldots, 9$. In other words, the mode $x_{i}$ is more severe than $x_{i+1}$.

The solution to this problem is obviously reduced to finding the extremum of the function $R_{Q}=\sum_{i=1}^{10} \beta_{i} \int_{0}^{\infty} y d Q\left(y / x_{i}\right)$. In this example $\xi$ and $\eta$ are assumed to be, respectively, the operating mode of the construction product and its moment of failure. Distributions $\mathrm{F}$ and $\Pi$ are defined by sets of probabilities $\alpha_{i}$ and $\beta_{i}, G(y)=P(\eta<\mathrm{y})=$ $1-e^{-\lambda y}$ while $y \geq 0$.

According to the theorem, we compose the probability ratio $\gamma(\mathrm{x})$ and we get:

$\gamma_{1}=\gamma_{2}=\gamma_{3}=\gamma_{4}=\gamma_{6}=\gamma_{8}=1, \gamma_{5}=\gamma_{10}=0,5, \gamma_{7}=\gamma_{9}=1,5$

because $\gamma_{1}=\gamma_{2}=\gamma_{3}=\gamma_{4}>\gamma_{5} ; \gamma_{6}<\gamma_{7}>\gamma_{8}$ и $\gamma_{8}<\gamma_{9}>\gamma_{10}$, then combining them into one group $\mathrm{x}_{1}, \mathrm{x}_{2}, \mathrm{x}_{3}, \mathrm{x}_{4}, \mathrm{x}_{6}, \mathrm{x}_{7}, \mathrm{x}_{8}, \mathrm{x}_{9}$, we get the following equation:

$$
\gamma^{(1)}(x)=\left\{\begin{array}{c}
1, x=x_{1}, \ldots, x_{7} \\
1,25, x=x_{8}, x_{9} \\
0,5, x=x_{10}
\end{array}\right.
$$

Because $\gamma_{1}^{(1)}=\gamma_{2}^{(1)}=\ldots=\gamma_{7}^{(1)}<\gamma_{8}^{(1)}=\gamma_{9}^{(1)}>\gamma_{10}^{(1)}$, we can combine all $\mathrm{x}_{\mathrm{i}}$ (but $\mathrm{x}_{10}$ ) into one group and construct the limiting probability ratio:

$$
\gamma^{(2)}(x)=\left\{\begin{array}{c}
\frac{9,5}{9}, x=x_{1}, \ldots, x_{9} \\
0,5, x=x_{10}
\end{array}\right.
$$

Then, using formula (1), we find the extreme distribution: 


$$
Q(y / x)=\left\{\begin{array}{c}
0, y<0 \\
\frac{1-\exp (-\lambda y)}{1-\exp (-\lambda \tilde{y})}, 0 \leq y<\tilde{y} \\
1, y \geq \tilde{y} \\
0, y<\tilde{y} \\
1-\exp (-\lambda(y-\tilde{y})), y \geq \tilde{y}
\end{array}\right\} x=x_{1}, \ldots, x_{9}
$$

where $\tilde{y}$ is determined from the equation $1-\exp (-\lambda \tilde{y})=0,9$, i.e. $\tilde{y}=-T \cdot \ln 0,1$.

After substituting $\mathrm{Q}$ into the expression of the function $R_{Q}$, we obtain $\mathrm{m}=0,872, \mathrm{~T}=872$ hours.

The absolute maximum $\mathrm{M}$ is calculated in the same way.

Omitting intermediate reasoning, we note that in this case the set $\left(x_{1}, x_{2}, \ldots, x_{10}\right)$ splits into three limiting groups: $X_{1}=\left(x_{1}, x_{2}, \ldots, x_{5}\right) ; X_{2}=\left(x_{6}, x_{7}\right) ; X_{3}=\left(x_{8}, x_{9}, x_{10}\right)$. So $\mathrm{M}=1,09, \mathrm{~T}=1090$ hours. Thus, the average time $R_{Q}$ of no-failure operation of a construction product under a given test mode will be concluded between 872 and 1090 hours.

This example shows the possibility of using this algorithm for a statistical assessment of the probable duration of a construction product according to the specified parameters.

Let's consider the application of this algorithm as applied to another operating mode.

Let the performance of a construction product be described by one technical parameter $\mathrm{x}[16,20]$, and as a result of acceptance and periodic tests of a certain batch of construction products, the corresponding distributions $F(x)$ and $G(t)$ are obtained. Moreover, it is known that $F(x)=P(X<x)$ is a normal distribution with parameters $\mu_{0}$ and $\sigma_{0}, G(t)=$ $P(\xi<t)=1-e^{-\lambda t}$, where $X$ is the initial value of the technical parameter, and $\xi$ is the moment of failure of a construction product.

It is required to find the minimum and maximum uptime of a new batch of construction products, in which the technical parameter is distributed according to the normal law with other parameters $\mu$ and $\sigma$. Applying the previously described algorithm to solve this problem, we obtain the values $m(\Pi, F, G)$ and $M(\Pi, F, G)$ for different values of $k=\sigma / \sigma_{0}$ $a=\left(\mu_{0}-\mu\right) / \sigma_{0}$, which are presented in the table 1 .

Table 1. Absolute maximum $M(\Pi, F, G)$ and absolute minimum $m(\Pi, F, G)$ values

\begin{tabular}{|c|c|c|c|c|c|c|c|}
\hline $\mathrm{k}^{\mathrm{a}}$ & $-0,5$ & $-0,3$ & $-0,1$ & 0 & 0,1 & 0,3 & 0,75 \\
\hline 0,1 & 1,3576 & 1,1210 & 1,9559 & 0,8245 & 0,7403 & 0,5924 & 0,4703 \\
& 0,7331 & 0,6578 & 0,7793 & 0,6962 & 0,6195 & 0,4842 & 0,3702 \\
\hline 0,2 & 1,3630 & 1,1174 & 0,9043 & 0,8098 & 0,7227 & 0,5778 & 0,4705 \\
& 0,1905 & 0,9761 & 0,7892 & 0,7058 & 0,6287 & 0,4932 & 0,3794 \\
\hline 0,3 & 1,3778 & 1,1177 & 0,8912 & 0,7908 & 0,7241 & 0,6118 & 0,5257 \\
& 1,2085 & 0,9934 & 0,8057 & 0,7216 & 0,6440 & 0,5070 & 0,3919 \\
\hline 0,4 & 1,4086 & 1,1267 & 0,8944 & 0,8232 & 0,7600 & 0,6566 & 0,5821 \\
& 1,2306 & 1,0167 & 0,8282 & 0,7437 & 0,6660 & 0,5266 & 0,4101 \\
\hline 0,5 & 1,4571 & 1,1512 & 0,9233 & 0,8549 & 0,7951 & 0,7018 & 0,6412 \\
& 1,2521 & 1,0433 & 0,8560 & 0,7709 & 0,6925 & 0,5514 & 0,4337 \\
\hline 0,6 & 1,4920 & 1,2035 & 0,9520 & 0,8855 & 0,8299 & 0,7483 & 0,7064 \\
& 1,2687 & 1,0694 & 0,8862 & 0,7965 & 0,7236 & 0,5821 & 0,4624 \\
\hline 0,7 & 1,5349 & 1,2758 & 0,9808 & 0,9164 & 0,8640 & 0,7998 & 0,7805 \\
& 1,2819 & 0,9292 & 0,9164 & 0,8343 & 0,7563 & 0,5514 & 0,4902 \\
\hline 0,8 & 1,5684 & 1,3502 & 1,0104 & 0,9458 & 0,8988 & 0,8601 & 0,8798 \\
& 1,2904 & 1,0694 & 0,9451 & 0,8655 & 0,7236 & 0,6516 & 0,4624 \\
\hline
\end{tabular}




\section{Conclusions}

The developed algorithm allows for a statistical assessment of the probable operation time of construction products and materials in cases when a number of initially specified parameters are known and their distribution function is determined for various modes.

The data obtained in table 1 is applicable for engineering calculations, used for construction product reliability determination in cases with the known technical parameters of that product.

\section{References}

1. L. N. Bolshev, E. A. Loginov, Probability theory and its application 10, 94-107 (1966)

2. A. N. Shiryaev, I. G. Ehrlich, P.A. Oskov, MCCME, 648 (2013)

3. N. M. Chiganova, Journal of Natural and Technical Sciences 6, 49-52 (2015)

4. L. N. Bolshev, E. V. Smirnov, Nauka, 416 (2012)

5. G.D. Kartashov, N.M. Chiganova, Journal of Mathematical Sciences 39, 2578-2588 (1987)

6. I. A. Ushakov, Journal of International Group on Reliability 1 (1), 6-19 (2007)

7. N. M. Chiganova, MATEC Web of Conferences 86, 02009 (2016)

8. B. V. Gnedenko, Radio I Svyaz, 376 (1983)

9. V. V. Medvedev, N. M. Chiganova, Scientific Review (Russia) 14, 232-236 (2015)

10. B.V. Gnedenko, J.K. Beljaev, A.D. Solov'ev, Nauka, 524 (2013)

11. N.M. Chiganova, Natural and Technical Sciences 6, 14-17 (2015)

12. V. Medvedev, A. Pustovgar, Applied Mechanics and Materials 725-726, 337-382 (2015)

13. B. V. Gnedenko, Yu. K. Belyaev, A. D. Soloviev, V. A. Kashtanov, Book House "LIBROKOM", 550 (2013)

14. G.D. Kartashov, Elements and schemes accelerated testing (monograph) (Knowledge, 1990)

15. I.A. Ushakov, Reliability: Theory \& Applications 2, 6-19 (2007)

16. G.D. Kartashov, Theory of Probability and its Applications 14(4), 595-611 (1969)

17. N.M. Sedjakin, Technical Cybernetics - USSR Academy of sciences proceedings 3 , 80-87 (1968)

18. A.I. Kubarev, I.Z. Aronov, O.I. Teskin et al., Standards Publishing House - USSR Gosstandart (1991)

19. L. V. Kir'yanova L.V., A. R. Usmanov, Vestnik MGSU 10, 88-94 (2012)

20. I. M. Ryzhik, I. S. Gradstein, BHV-Peterburg, 1182 (2011) 